\title{
Fermi-LAT observation of Intermediate Synchrotron Peaked blazars
}

\author{
Claudia Monte \\ Dipartimento di Fisica "M. Merlin" dell'Università e del Politecnico di Bari, I-70126, Bari, Italy \\ Istituto Nazionale di Fisica Nucleare, Sezione di Bari, I-70126 Bari, Italy \\ E-mail: claudia.monte@ba.infn. it \\ on behalf of the Fermi Large Area Telescope Collaboration
}

\begin{abstract}
After the first year of scientific activity with the Large Area Telescope (LAT), i.e. the primary instrument onboard Fermi, the 1st LAT Active Galactic Nuclei Catalog (1LAC) has been published. Among the 1LAC blazars, a particular and interesting group of objects is the one composed by Intermediate Synchrotron Peaked (ISP) blazars, i.e. sources for which the synchrotron emission peak in the Spectral Energy Distribution (SED) is located at intermediate frequencies (from $10^{14} \mathrm{~Hz}$ to $10^{15} \mathrm{~Hz}$ ). These objects are expected to have their SED high-energy peak centered on the Fermi LAT band (from $20 \mathrm{MeV}$ to $300 \mathrm{GeV}$ ). The brightest 1LAC ISP candidates have been selected and they have been analyzed covering a period of 22 months of Fermi LAT gamma-ray data in order to investigate their spectral features and to characterize the temporal evolution of their gamma-ray spectra. The preliminary results of the spectral analysis, performed with different methods, of some of these objects will be presented.
\end{abstract}

25th Texas Symposium on Relativistic Astrophysics - TEXAS 2010

December 06-10, 2010

Heidelberg, Germany 


\section{Introduction}

The Gamma-ray Large Area Space Telescope was launched on June 11, 2008 into a low Earth circular orbit at an altitude of $550 \mathrm{~km}$ and an inclination of $25.6^{\circ}$. It began its scientific operation two months later, and shortly thereafter, it was renamed the Fermi Gamma-ray Space Telescope. The Large Area Telescope (LAT), the main instrument onboard Fermi, is a pair production telescope with large effective area $\left(\sim 8000 \mathrm{~cm}^{2}\right.$, on axis for $\left.E>1 \mathrm{GeV}\right)$ and field of view $(\sim 2.4 \mathrm{sr}$ at $1 \mathrm{GeV}$ ), sensitive to gamma-rays in the energy range from $20 \mathrm{MeV}$ to more than $300 \mathrm{GeV}$ [1].

One of the major scientific goals of the Fermi mission is to investigate high-energy emission in active galactic nuclei (AGN). Although AGNs are probably one of the better known class of gamma-ray emitting objects, there are many questions that are yet unanswered, as the mechanisms by which the particles are accelerated, the precise site of the gamma-ray emission and the origin of AGN variability [2].

In the unification scenario [3] AGN are described with a central supermassive black hole whose gravitational potential energy is the ultimate source of the AGN luminosity. An accretion disk, providing fuel for the supermassive black hole, and broad- and narrow-line emission regions surround the black hole itself. The optical and ultaviolet radiation coming from the broad-line region is obscured along some lines of sight by a torus of gas and dust well outside the accretion disk and the broad-line region. The radio-loud AGNs are characterized by the presence of jets of collimated plasma ejected with relativistic speeds transverse to the plane of the accretion disk. Such jets are very weak or absent in radio-quiet AGNs. Radio-loud and radio-quiet AGNs viewed at different angles will be assigned to different classes. In particular, blazars are radio-loud AGNs with their relativistic radio jet pointing in a direction that is closely aligned to the observer line of sight.

Of the 1043 high-latitude $\left(|b|>10^{\circ}\right)$, gamma-ray sources in the Fermi Large Area Telescope First Source Catalog (1FGL) [4], detected in 11 months (from August 4, 2008 to July 4, 2009)

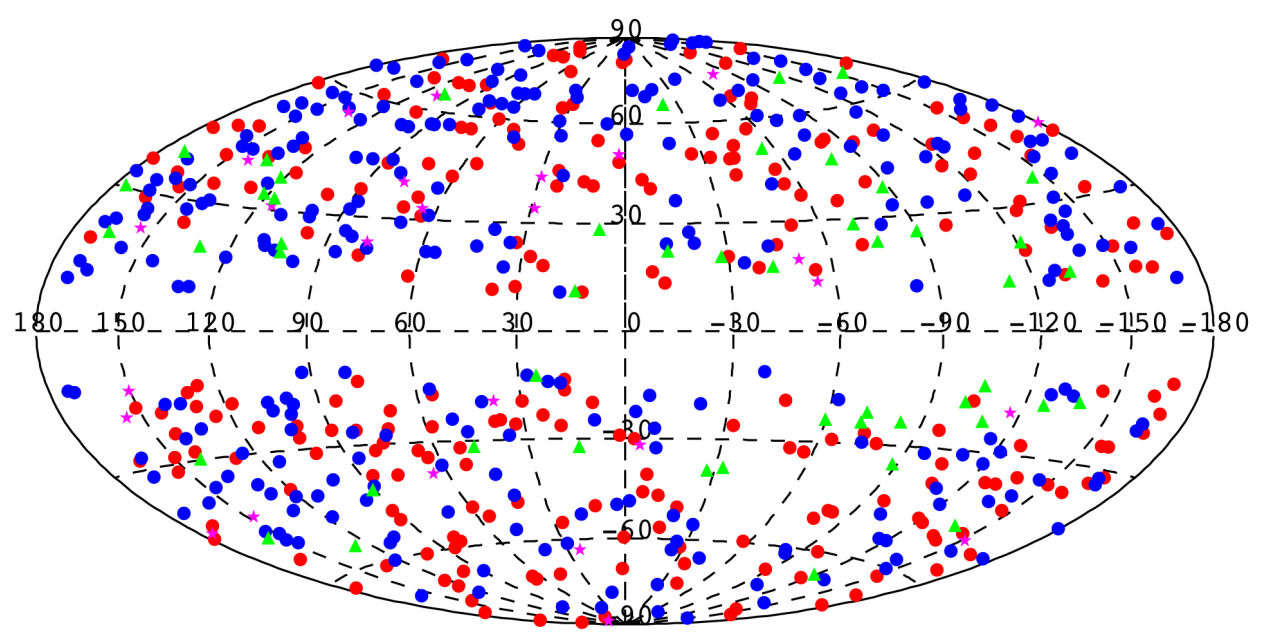

Figure 1: Locations of the associated sources in the 1LAC. The 1LAC includes 300 BL Lacs blazars (blue full circles), 296 FSRQs blazars (red full circles), 41 AGNs of other types, including radio galaxies (magenta stars) and 72 AGNs of unknown type (green triangles) [2]. 
of scientific data collected with standard sky-survey observation, the 1st LAT Catalog of Active Galactic Nuclei (1LAC) [2], includes 671 sources detected with a test statistic greater than 25 and statistically associated with 709 AGNs (due to some multiple associations). The 1LAC is mainly composed of blazars; in fact it includes 300 BL Lacertae (BL Lacs) blazars and 296 Flat Spectrum Radio Quasar (FSRQs) blazars, as shown in figure 1.

\section{The SED-based classification of blazars}

Blazars are highly variable objects, therefore simultaneous observations in different energy bands are essential to understand the emission processes that take place in these objects. For 48 blazars belonging to the LBAS (LAT Bright AGN Sample) [5], the first three months (from August 4, 2008 to October 31, 2008) of Fermi LAT data were combined with simultaneous observations at other wavelengths, in order to assemble high-quality and quasi-simultaneous Spectral Energy Distributions (SEDs) [6]. All the SEDs clearly showed the typical two-bump signature with the two energy peaks attributed respectively to synchrotron (low energy peak) and inverse-Compton (high energy peak ) emission. $\mathrm{T}$ he determination of $v_{\text {peak }}^{S}$ (the frequency of the low energy synchrotron peak position) for a large number of blazars has permitted the development of a new SED-based classification [6] for all blazars, shown schematically in figure 2.

- Low Synchrotron Peaked (LSP) blazars are sources with the synchrotron peak at low energy (i.e. in the far IR or IR band or $v_{\text {peak }}^{S} \leq 10^{14} \mathrm{~Hz}$ );

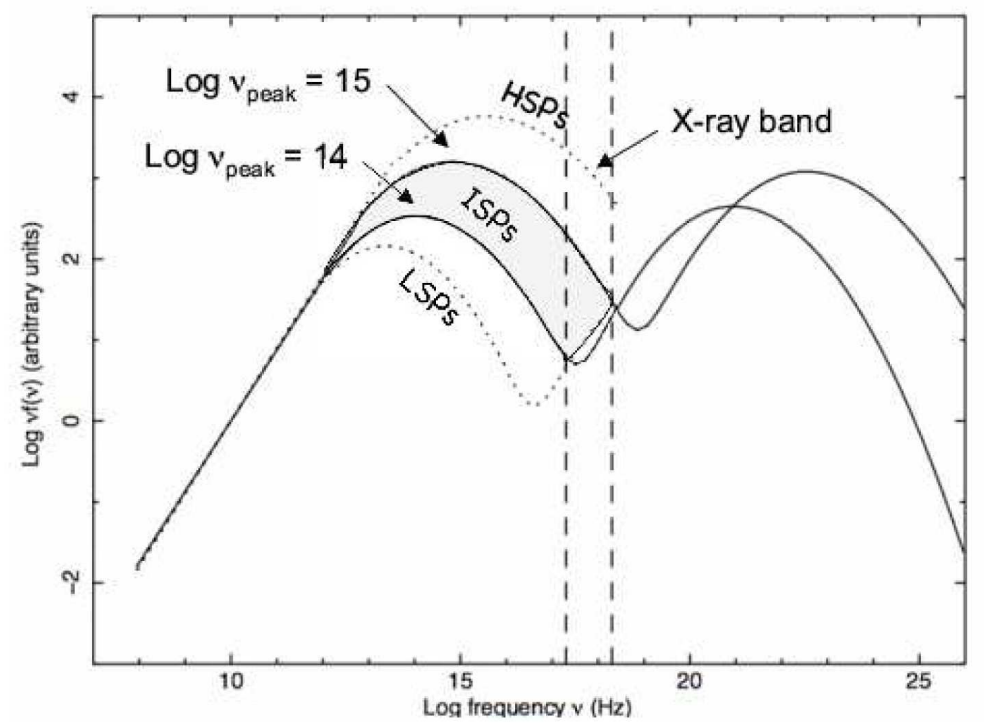

Figure 2: The definition of different blazar types based on the peak of the synchrotron component $\left(v_{\text {peak }}^{S}\right)$ in their SED. Low Synchrotron Peaked blazars, or LSP (lower dotted line). Intermediate Synchrotron Peaked sources (central continuous line with the SED peak within the grey area). High Synchrotron Peaked blazars, or HPS (upper dotted line). See section 2 for LSP, ISP and HSP definitions [6]. 
- High Synchrotron Peaked (HSP) blazars have the synchrotron peak at UV or higher energies $\left(v_{\text {peak }}^{S} \geq 10^{15} \mathrm{~Hz}\right)$

- Intermediate Synchrotron Peaked (ISP) blazars are sources for which the synchrotron emission peaks at intermediate frequencies (from $10^{14} \mathrm{~Hz}$ to $10^{15} \mathrm{~Hz}$ ). For these objects the inverse-Compton (high energy) peak is expected to be exactly inside the Fermi LAT energy band.

\section{Fermi LAT ISP blazars study}

Looking at the gamma-ray spectra of 1LAC (first LAT AGN Catalog) blazars [2], a sample of about 30 sources, including Flat Spectrum Radio Quasar (FSRQs) and BL Lac objects, showing the SED high energy peak in the Fermi LAT energy band, has been selected.

For these sources, that are ISP candidates, 22 months (from August 4, 2008 to June 4, 2010) of Fermi LAT data have been analyzed in the energy range from $100 \mathrm{MeV}$ to $300 \mathrm{GeV}$, considering a Region of Interest (RoI) of $15^{\circ}$ around the source under study and using different methods. In the first case, the whole energy band has been divided into 3 equal logarithmically spaced energy bins per decade and a maximum likelihood fit has been used to calculate the flux in each band: a Power Law parametric model with Spectral Index fixed at 2, has been assumed in each individual energy bin both for the source spectrum and for the background components. The flux of the source in all energy bins has beenevaluated, requiring in each bin a Test Statistic (TS) ${ }^{1}$ greater than 10 . When the $T S \leq 10$, the flux values have been replaced by $2 \sigma$ upper limits ${ }^{2}$. In the second and third method of analysis, a maximum likelihood fit has been performed in the whole energy band (from $100 \mathrm{MeV}$ to $300 \mathrm{GeV}$ ) modeling the source under investigation respectively with a Power Law (PL) or with a Log Parabola (LP). For all three previous described methods, the maximum likelihood analysis has been performed using the Fermi science tool gtlike ${ }^{3}$.

Once the differential flux $\Phi(E)$ has been evaluated, the corresponding SED has been obtained by multiplying the differential flux by the square of the energy:

$$
v F(v)=E^{2} \Phi(E)
$$

where $E=h v$.

In figures 3 and 4 , the gamma-ray SED obtained with the different methods respectively for the sources $4 \mathrm{C}+55.17\left(1 \mathrm{FGL}\right.$ J0957.7 $\left.+5523, R A=149.432^{\circ}, D E C=55.392^{\circ}\right)$ and $\mathrm{B} 21229+29$

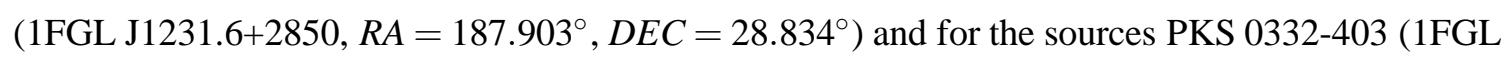
$\left.\mathrm{J} 0334.2-4010, R A=53.553^{\circ}, D E C=-40.168^{\circ}\right)$ and CGRaBS J2345-1555 (1FGL J2344.6-1554, $R A=356.168^{\circ}, D E C=-15.907^{\circ}$ ) are shown. The blue points represent the results of the gtlike analysis performed in each energy bin, the green continuous line and the magenta dashed line represent the results of the gtlike analysis in the whole energy band obtained modeling the source under investigation respectively with a PL and with a LP.

\footnotetext{
${ }^{1}$ The Test Statistic is defind as: $T S=-2 \times\left(\log \left(L_{0}\right)-\log \left(L_{1}\right)\right)$ with $L_{0}$ and $L_{1}$ respectively the likelihood results for models with and without the source under investigation.

${ }^{2}$ The $2 \sigma$ Upper Limit has been derived by finding the flux value for which $T S=4$.

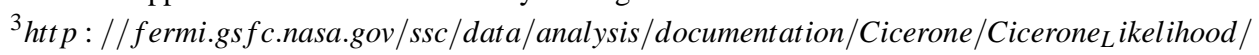



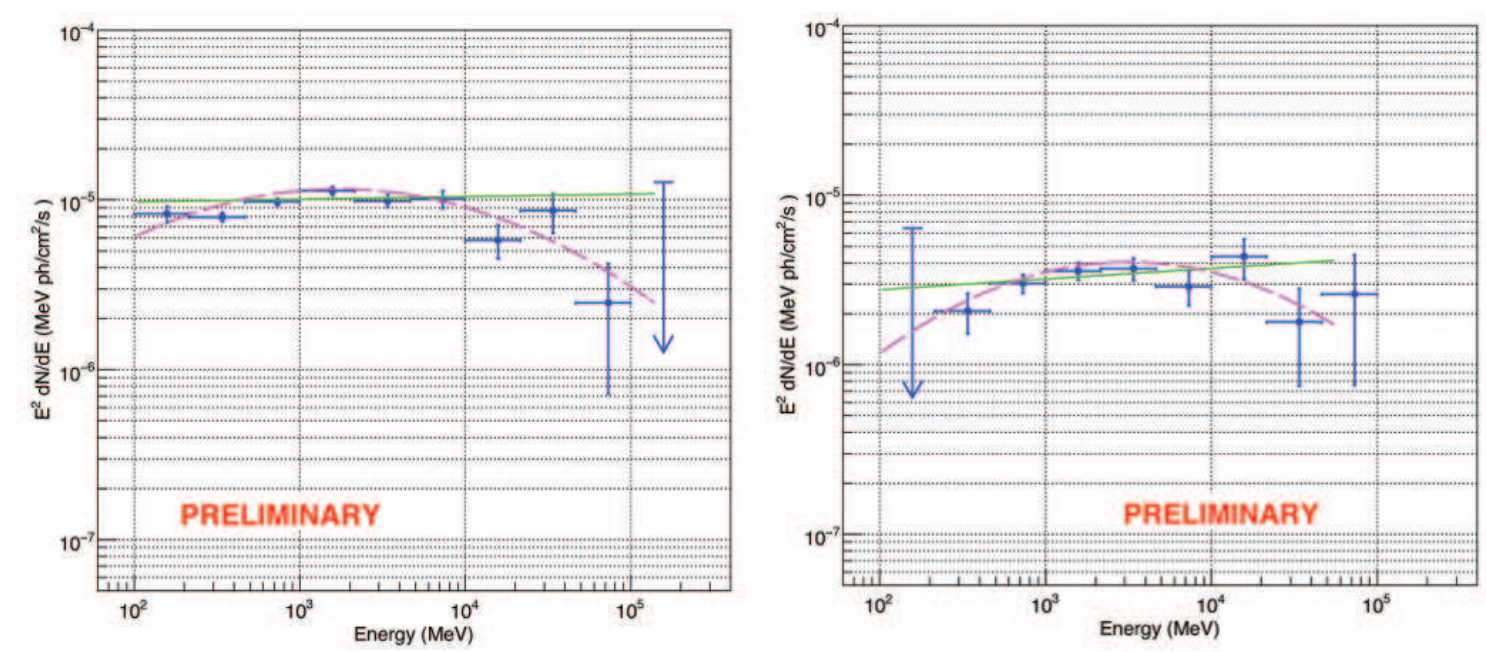

Figure 3: The gamma-ray SED of 1FGL J0957.7+5523 $=4 \mathrm{C}+55.17$ (left) and 1FGL J1231.6+2850= B2 1229+29 (right). Blue points : gtlike analysis performed in each energy bin. Green continuous line: gtlike analysis in the whole energy band obtained modeling the source under investigation with a Power Law. Magenta dashed line: gtlike analysis in the whole energy band obtained modeling the source under investigation with a Log Parabola.

It is possible to calcualte the LogLike value for both the gtlike fits in the whole energy band and than it is possible to compute the TSCurve index, defined as:

$$
\text { TSCurve }=2 \times(\operatorname{loglike}(L P)-\log \text { like }(P L))
$$

that quantifies the improvement of the Log Parabola fit with respect to the Power law fit. For all the sources in our sample, the TSCurve value is greater than 0 . In particular, for the sources shown here, the TSCurve values are: TSCurve $=31.0$ for the $4 \mathrm{C}+55.17$ source, TSCurve $=10.8$ for the B2 1229+29 source, TSCurve $=9.6$ for the PKS 0332-403 source and TSCurve $=15.4$ for the CGRaBS J2345-1555 source. So, for all sources here, the TSCurve value is greater than 9, corresponding to a significance of $3 \sigma$. For almost all sources in our selected sample, the TSCurve value is greatrer than 9, i.e. it is likely (with a significance of $\sim 3 \sigma$ ) the Log Parabola is a better representation of the spectrum of these kind of sources than a simple Power Law.

Assuming that the Log Parabola is the right function to describe the spectra of our sources, and assuming that the differential flux for these sources can be written as:

$$
\frac{d N}{d E}=N_{0}\left(\frac{E}{E_{b}}\right)^{-\left(\alpha+\beta \log \left(\frac{E}{E_{b}}\right)\right)}
$$

where $N_{0}$ is the normalization parameter and $E_{b}$ is the Energy break value, fixed at $1 \mathrm{GeV}$ for our sources, it is possible to calculate the Log Parabola peak energy [7], using the following formula:

$$
E_{p}=E_{b} 10^{\frac{(2-\alpha)}{2 \beta}}
$$



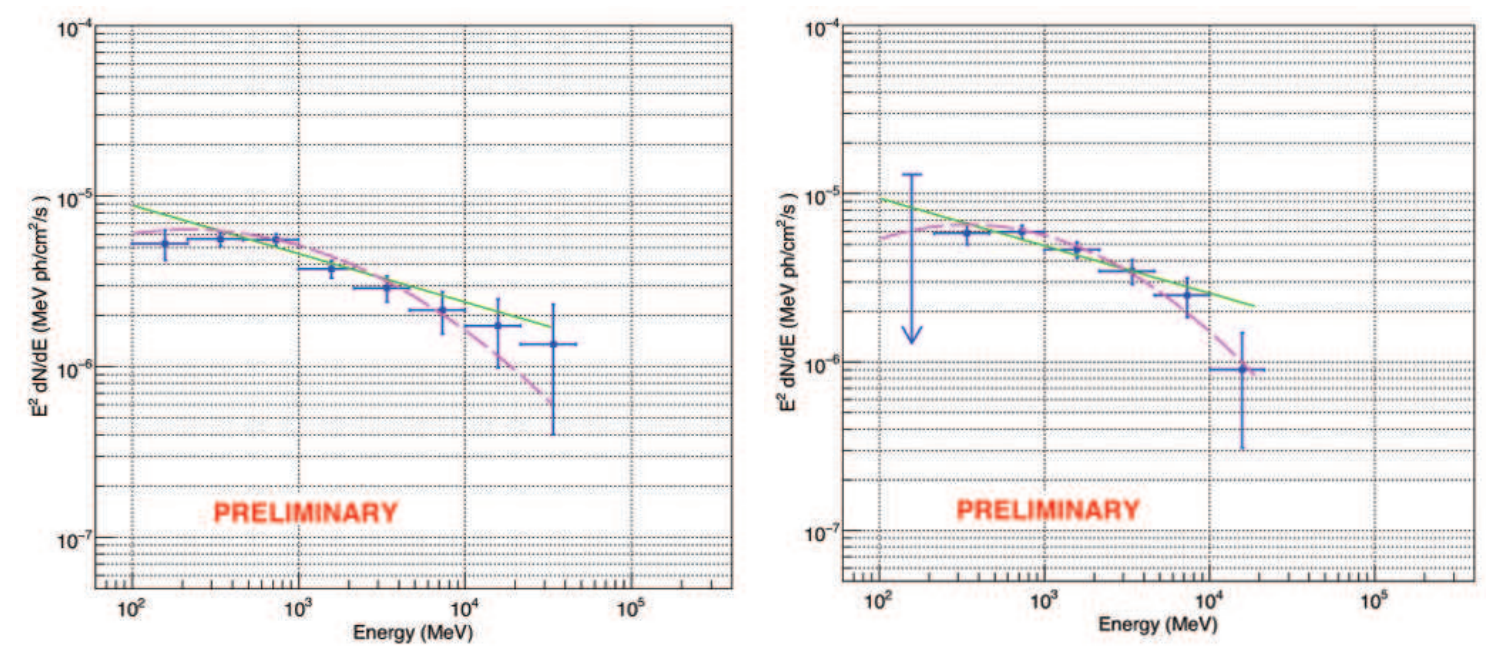

Figure 4: The gamma-ray SED of 1FGL J0334.2-4010 = PKS 0332-403 (left) and 1FGL J2344.6-1554= CGRaBS J2345-1555 (right). Blue points : gtlike analysis performed in each energy bin. Green continuous line: gtlike analysis in the whole energy band obtained modeling the source under investigation with a Power Law. Magenta dashed line: gtlike analysis in the whole energy band obtained modeling the source under investigation with a Log Parabola.

\section{Conclusions}

A sample of about 30 ISP candidates has been selected from the 1LAC. Preliminary spectral analysis performed over a period of 22 months seems to show that the Log Parabola function can be used to model the shape of the SED high-energy peak in the gamma-ray energy range for these sources. Using the Log Parabola fit spectral parameters the position of the peak has been calculated and for all sources it has been found that it is placed inside the Fermi LAT energy band (below or above $1 \mathrm{GeV}$, depending on the source). The analysis is still ongoing in order to find any other spectral features and to investigate about variability properties for this set of sources. In fact the gamma-ray radiation emitted in the Fermi LAT energy band, could carry on information mainly on the synchrotron and the inverse Compton emission mechanisms, probably leading to the distinction between synchrotron self Compton (SSC) and external radiation Compton (ERC) dominated sources.

\section{Acknowledgments}

The Fermi LAT Collaboration acknowledges support from a number of agencies and institutes for both development and the operation of the LAT as well as scientific data analysis. These include NASA and DOE in the United States, CEA/Irfu and IN2P3/CNRS in France, ASI and INFN in Italy, MEXT, KEK, and JAXA in Japan, and the K. A. Wallenberg Foundation, the Swedish Research Council and the National Space Board in Sweden. Additional support from INAF in Italy and CNES in France for science analysis during the operations phase is also gratefully acknowledged. 


\section{References}

[1] W.B. Atwood et al.,ApJ, 697, 1071 (2009)

[2] A.A. Abdo et al., ApJ, 715, 429 (2010)

[3] C.M. Urry \& P. Padovani, PASP, 107, 803 (1995)

[4] A.A. Abdo et al., ApJS, 188, 405 (2010)

[5] A.A. Abdo et al., ApJ, 700, 597 (2009)

[6] A.A. Abdo et al., ApJ 716, 30 (2010)

[7] E. Massaro et al., $A \& A$ 448, 861 (2006) 